\title{
ON COMPACTNESS OF COMPOSITION OPERATORS IN HARDY SPACES OF SEVERAL VARIABLES
}

\author{
SONG-YING LI AND BERNARD RUSSO
}

(Communicated by Palle E. T. Jorgensen)

\begin{abstract}
Characterizations of compactness are given for holomorphic composition operators on Hardy spaces of a strongly pseudoconvex domain.
\end{abstract}

\section{INTRODUCTION}

Let $\Omega$ be a bounded domain in $\mathbf{C}^{n}$ with $C^{1}$ boundary. Let $\varphi$ be a holomorphic mapping from $\Omega$ to $\Omega$. The composition operator $C_{\varphi}$ is defined formally as follows: $C_{\varphi}(u)(z)=u(\varphi(z))$ for all $z \in \Omega$ and any function $u$ on $\Omega$. The study of such holomorphic composition operators has been active since the early 1970s (see Cowen [5] for details in the case of one variable). In the case of several complex variables, counterexamples have been constructed by several authors showing that composition operators can be unbounded on $\mathscr{H}^{2}\left(B_{n}\right)$, where $B_{n}$ is the unit ball in $C^{n}$ (see, for example, Cima and Wogen [1], Wogen's survey paper [24], and the references therein). In this paper, we are concerned with compactness of composition operators. It was proved by Shapiro and Taylor [22] that $C_{\varphi}: \mathscr{H}^{p}\left(B_{1}\right) \rightarrow \mathscr{H}^{p}\left(B_{1}\right)$ is compact for one $p \in(0, \infty)$ if and only if it is compact on $\mathscr{H}^{p}\left(B_{1}\right)$ for all $p \in(0, \infty)$. There is a characterization of compactness for $C_{\varphi}: \mathscr{H}^{p}\left(B_{1}\right) \rightarrow \mathscr{H}^{p}\left(B_{1}\right)$ in terms of the Nevanlinna counting function, given by Shapiro [19]. Another characterization of compactness can be formulated in terms of a Carleson measure condition for the pullback measure $d \nu_{\varphi}$ (see [16] for the case of the unit ball in $\mathbf{C}^{n}$ ). This theorem has also been proved for the unit polydisk in $\mathbf{C}^{n}$ in [10].

More recently, Sarason [20] proved that weak compactness and norm compactness for a composition operator on the Hardy space $\mathscr{H}^{1}\left(B_{1}\right)$ are equivalent. He found it more natural to consider real Hardy space on $\partial B_{1}$ rather than holomorphic Hardy space. Using the duality theorem of Fefferman on $H^{1}$, Sarason proved that norm compactness on $H^{1}\left(\partial B_{1}\right)$ is equivalent to $C_{\varphi}^{*}\left(B M O\left(\partial B_{1}\right)\right) \subset$ $V M O\left(\partial B_{1}\right)$. Later, Shapiro and Sundberg [21] proved that compactness of $C_{\varphi}$ on $\mathscr{H}^{2}\left(B_{1}\right)$ is equivalent to compactness on $L^{1}\left(\partial B_{1}\right)$, where the composition operator is now viewed as acting on the Poisson integral of functions in $L^{1}\left(\partial B_{1}\right)$.

Received by the editors April 12, 1993.

1991 Mathematics Subject Classification. Primary 47B38, 32A35.

(C) 1994 American Mathematical Society $0002-9939 / 94 \$ 1.00+\$ .25$ per page 
The main purpose of this paper is to generalize the above theorems of Sarason and Shapiro-Taylor from the unit disc to strongly pseudoconvex domains $\Omega$ in $\mathbf{C}^{n}$. Some of our results are known in the case of the unit ball $B_{n}$ in $\mathbf{C}^{n}$ [26]. Our formulation differs slightly from that of Sarason [20]. This is explained in a remark following the statement of the main theorem in $\S 2$.

\section{NOTATION AND STATEMENT OF THE MAIN THEOREM}

Let $\Omega$ be a bounded strongly pseudoconvex domain with $C^{2}$ boundary. We define a continuous function $d$ on $\partial \Omega$ as follows. For $x \in \partial \Omega$, let $\pi_{x}$ denote the complex tangent plane at $x$. For $t>0, A_{x, t}$ denotes the set of points in $\mathbf{C}^{n}$ at distance $\leq t$ from the ball in the plane $\pi_{x}$ with center at $x$ and radius $\sqrt{t}$. Let $B_{x, t}=A_{x, t} \cap \partial \Omega$. Then set

$$
d(x, y)=\inf \left\{t>0 ; y \in B_{x, t}, x \in B_{y, t}\right\} .
$$

It is known that $d$ defined above is a quasimetric on $\partial \Omega$ (see, for example, $[23,12])$, i.e., $d$ is a continuous function from $\partial \Omega \times \partial \Omega$ to $\mathbf{R}^{+}$satisfying the usual requirements for a topological metric except that the triangle inequality is replaced by

$$
d(x, z) \leq C(d(x, y)+d(y, z)), \quad x, y, z \in \partial \Omega .
$$

Let $\Omega$ be a smoothly bounded domain in $\mathbf{C}^{n} \quad(n \geq 2)$. We shall use $r(z)$ to denote the distance function of $z \in \Omega$ to $\partial \Omega$. We define $\mathscr{H}^{p}(\Omega) \quad(0<p<\infty)$ to be the usual Hardy space of holomorphic function on $\Omega$ (see [12, Chapter 8]). We may identify it with a closed subspace of $L^{p}(\partial \Omega)$ by passing to the (almost everywhere) radial limit function $\tilde{f}$ on $\partial \Omega$. Let $d$ be a quasimetric on $\partial \Omega$. Then $B M O(\partial \Omega) \subset L^{1}(\partial \Omega)$ is defined in terms of the quasimetric $d$ and the surface measure $\sigma$ on $\partial \Omega$ as follows. The seminorm on $B M O$ is defined for $g \in L^{1}(\partial \Omega)$ by

$$
\|g\|_{B M O}=\sup _{x, r} M O(g)(x, r),
$$

where

$$
M O(g)(x, r)=\frac{1}{|B(x, r)|} \int_{B(x, r)}\left|g(t)-g_{B(x, r) \mid}\right| d \sigma(t) .
$$

Here the balls $B(x, r)$ (called nonisotropic) are defined using the quasimetric, $g_{B(x, r)}$ is the average of $g$ over the ball, $d \sigma$ is $(2 n-1)$-dimensional surface measure on the boundary of $\Omega$, and $|B(x, r)|=\sigma(B(x, r))$. We say a function $f \in L^{1}(\partial \Omega)$ belongs to $V M O(\partial \Omega)$ if $f \in B M O(\partial \Omega)$ and $\sup _{x \in \partial \Omega} M O(f)(x, r) \rightarrow 0$ as $r \rightarrow 0$. Now $B M O A(\Omega)$ denotes the space of functions in $\mathscr{H}^{1}(\Omega)$ whose boundary values $\tilde{f}$ are in $B M O(\partial \Omega)$, with norm $\|f\|_{*}=\|\tilde{f}\|_{B M O}$; and $V M O A(\Omega)=B M O A(\Omega) \cap V M O(\partial \Omega)$. It is easy to prove that $B M O A(\Omega)$ is a closed subspace of $B M O(\partial \Omega)$, and that $V M O A(\Omega)$ is a closed subspace of $V M O(\partial \Omega)$.

A measurable function $a$ on $\partial \Omega$ is said to be an atom if either $a$ is bounded on $\partial \Omega$ and $|a|$ does not exceed $1 /|\partial \Omega|$ or there is a point $z_{0} \in \partial \Omega$ and $r>0$ such that $a$ is supported on $B\left(z_{0}, r\right)$ and satisfies the following conditions:

$$
|a(z)| \leq \frac{1}{\left|B\left(z_{0}, r\right)\right|}, \quad \int_{\partial \Omega} a(z) d \sigma(z)=0 .
$$


We say a function $u$ belongs to $H^{1}(\partial \Omega)$ (real $H^{1}$ ) if there is a sequence of atoms $\left\{a_{k}\right\}$ and a sequence of numbers $\left\{\lambda_{k}\right\} \in l^{1}$ such that $u=\sum_{k=1}^{\infty} \lambda_{k} a_{k}$ in the sense of distributions. The norm of $u \in H^{1}(\partial \Omega)$ is defined as follows:

$$
\|u\|_{H^{1}}=\inf \left\{\sum_{k=1}^{\infty}\left|\lambda_{k}\right|: u=\sum_{k=1}^{\infty} \lambda_{k} a_{k}\right\} .
$$

The duality theorem of $B M O$ and $H^{1}$ has been shown by Coifman and Weiss in [2] for a general space of homogeneous type. As a special case of their theory, we state:

Theorem A. (i) $H^{1}(\partial \Omega)^{*}=B M O(\partial \Omega)$, and

(ii) $V M O(\partial \Omega)^{*}=H^{1}(\partial \Omega)$.

Let $P: L^{2}(\partial \Omega) \rightarrow \mathscr{H}^{2}(\Omega)$ be the orthogonal projection, that is, the Szegö projection with Szegö kernel $S(z, w)$. The relations between real $H^{1}$ and holomorphic $\mathscr{H}^{1} \Omega$ ) are given in [13] and [14] as follows:

Theorem B. Let $\Omega$ be a bounded strongly pseudoconvex domain in $\mathbf{C}^{n}$ or a pseudoconvex domain of finite type in $\mathbf{C}^{2}$ with smooth boundary. Then:

(i) $P\left(H^{1}(\partial \Omega)\right)=\mathscr{H}^{1}(\partial \Omega)$;

(ii) $\mathscr{H}^{1}(\Omega)^{*}=B M O A(\Omega), P(B M O(\partial \Omega))=B M O A(\Omega)=P\left(L^{\infty}(\partial \Omega)\right)$;

(iii) $V M O A(\partial \Omega)^{*}=\mathscr{H}^{1}(\Omega), P(V M O(\partial \Omega))=V M O A(\Omega)=P(C(\partial \Omega))$.

Let

$$
P_{0}(z, w)=S(z, z)^{-1}|S(z, w)|^{2}
$$

be the Poisson-Szegö kernel on $\Omega \times \partial \Omega$. The composition operator $C_{\varphi}$ extends to functions in $L^{1}(\partial \Omega)$ as follows: for $u \in L^{1}(\partial \Omega)$,

$$
C_{\varphi}(u):=C_{\varphi}\left(P_{0}(u)\right)=P_{0}(u) \circ \varphi,
$$

where for $z \in \Omega$

$$
P_{0}(u)(z)=\int_{\partial \Omega} u(w) P_{0}(z, w) d \sigma(w) .
$$

Let $z \in \partial \Omega$ and $r>0$. We shall use $C(z, r)$ to denote the Carleson region:

$$
C(z, r)=\{w \in \Omega: \pi(w) \in B(z, r), r(w)<r\},
$$

where $\pi(w)$ is the normal projection of $w$ to $\partial \Omega$. Let $\mathscr{M}(\bar{\Omega})$ denote the space of all complex Borel measures over $\bar{\Omega}$. Corresponding to $\mu \in \mathscr{M}(\bar{\Omega})$, we define the following function on $(0,1)$ :

$$
g_{\mu}(r)=\sup _{z \in \partial \Omega}\left\{\frac{|\mu|(C(z, r))}{|B(z, r)|}\right\} .
$$

Let $B C M(\Omega)$ denote the space of all Carleson measures over $\bar{\Omega}$, i.e., $d \mu \epsilon$ $B C M(\Omega)$ if $d \mu \in \mathscr{M}(\bar{\Omega})$ and $g_{\mu} \in L^{\infty}(0,1)$. By definition, the measure $d \mu \in \mathscr{M}(\bar{\Omega})$ belongs to $V C M(\Omega)$ if $d \mu \in B C M(\Omega)$ and $\lim _{r \rightarrow 0^{+}} g_{\mu}(r)=0$.

The pullback measure $d \nu_{\varphi}$ will play an important technical role in our proofs. Recall that to define it, one first extends $\varphi$ to $\bar{\Omega}$ by passing to the radial limits almost everywhere on $\partial \Omega$ (see [12]). Denoting this extension by $\varphi$ too, $d \nu_{\varphi}$ is the measure defined on $\bar{\Omega}$ by

$$
\nu_{\varphi}(E)=\sigma\left(\varphi^{-1}(E) \cap \partial \Omega\right) .
$$

Now we are ready to state our main theorem. 
Theorem 1. Let $\Omega$ be a bounded strongly pseudoconvex domain in $\mathbf{C}^{n}$ with smooth boundary. Let $\varphi: \Omega \rightarrow \Omega$ be a holomorphic mapping. Then the following statements are equivalent:

(i) $C_{\varphi}^{*}: L^{\infty}(\partial \Omega) \rightarrow V M O(\partial \Omega)$ is bounded; that is, $C_{\varphi}: H^{1}(\partial \Omega) \rightarrow L^{1}(\partial \Omega)$ and the range of $C_{\varphi}^{*}$ is contained in $V M O(\partial \Omega)$.

(ii) $C_{\varphi}: H^{1}(\partial \Omega) \rightarrow L^{1}(\partial \Omega)$ is compact.

(iii) $C_{\varphi}: \mathscr{H}^{1}(\Omega) \rightarrow \mathscr{H}^{1}(\Omega)$ is compact.

(iv) $C_{\varphi}: \mathscr{H}^{p}(\Omega) \rightarrow \mathscr{H}^{p}(\Omega)$ is compact for some $0<p<\infty$.

(v) $C_{\varphi}: L^{p}(\partial \Omega) \rightarrow L^{p}(\partial \Omega)$ is compact for some $1<p<\infty$.

(vi) $d \nu_{\varphi} \in V C M(\Omega)$.

(vii) $C_{\varphi}: \mathscr{H}^{p}(\Omega) \rightarrow \mathscr{H}^{p}(\Omega)$ is compact for all $0<p<\infty$.

Remark. Statement (ii) is slightly different from the compactness of $C_{\varphi}$ on $H^{1}(\partial \Omega)$. In the case $n=1$ and $\Omega=B_{1}$, a short argument using the Hilbert transform proves that if $C_{\varphi}: H^{1}(\partial \Omega) \rightarrow L^{1}(\partial \Omega)$ is compact, then it is compact on $H^{1}(\partial \Omega)$ (see [20] for detail). For $n>1$, estimating the $H^{1}(\partial \Omega)$ norm is much more complicated. A similar singular integral characterization for $H^{1}\left(\partial B_{n}\right)$ has been given by Christ and Geller in [3]. Although this may be the right tool to use here, it turns out to be very technical. For simplicity, we state and prove Theorem 1 as above.

\section{Preliminary Results}

In this section, we prove some steps in our main theorem. First, let us introduce a fundamental criteria for boundedness and compactness of composition operators. The boundedness part of the following theorem is well known (for example, see [24]).

Theorem 3.1. Let $\Omega$ be a bounded strongly pseudoconvex domain in $\mathbf{C}^{n}$ with smooth boundary. Let $\varphi: \Omega \rightarrow \Omega$ be a holomorphic mapping. The following statements hold:

(i) For $0<p<\infty, C_{\varphi}: \mathscr{H}^{p}(\Omega) \rightarrow \mathscr{H}^{p}(\Omega)$ is bounded if and only if $d \nu_{\varphi} \in B C M(\Omega)$.

(ii) For $0<p<\infty, C_{\varphi}: \mathscr{H}^{p}(\Omega) \rightarrow \mathscr{H}^{p}(\Omega)$ is compact if and only if $d \nu_{\varphi} \in V C M(\Omega)$.

(iii) $C_{\varphi}: H^{1}(\partial \Omega) \rightarrow L^{1}(\partial \Omega)$ is compact if and only if $d \nu_{\varphi} \in V C M(\Omega)$.

(iv) For $1<p<\infty, C_{\varphi}: L^{p}(\partial \Omega) \rightarrow L^{p}(\partial \Omega)$ is compact if and only if $d \nu_{\varphi} \in V C M(\Omega)$.

Remark. This theorem can be extended to a pseudoconvex domain of finite type in $C^{2}$ with $C^{\infty}$ boundary by using results in $[14,7,17]$.

Note that the equivalence of (ii) through (vii) in Theorem 1 is contained in Theorem 3.1.

In order to prove Theorem 3.1, let us first record the following result, whose statement and proof can be found in [15] for $\Omega=B_{N}$. For the general case, the proof is still elementary, so we omit the details here.

Proposition 3.2. Let $\Omega$ be a bounded domain in $\mathbf{C}^{n}$, let $\varphi: \Omega \rightarrow \Omega$ be a holomorphic mapping, and suppose $0<p<\infty$. Then $C_{\varphi}: \mathscr{H}^{p}(\Omega) \rightarrow \mathscr{H}^{p}(\Omega)$ is compact if and only if for each bounded sequence $\left\{u_{n}\right\}$ in $\mathscr{H}^{p}(\Omega)$ which 
converges to 0 on compacta on $\Omega$ it follows that $C_{\varphi}\left(u_{n}\right)$ converges to 0 in $\mathscr{H}^{p}(\Omega)$-metric.

We now turn to the proof of Theorem 3.1. In the rest of this section $d \nu$ will denote $d \nu_{\varphi}$.

Proof of Theorem 3.1. For completeness, we present a proof of (i). Let $u \in$ $\mathscr{H}^{p}(\Omega)$. Then, with $\Omega_{\epsilon}:=\{z \in \Omega: d(z, \partial \Omega)>\epsilon\}$, we have

$$
\begin{aligned}
\left\|C_{\varphi}(u)\right\|_{\mathscr{R} p(\Omega)}^{p} & =\sup _{0<\epsilon<<1}\left\{\int_{\partial \Omega_{\epsilon}}\left|C_{\varphi}(u)(z)\right|^{p} d \sigma_{\epsilon}(z)\right\} \\
& =\sup _{0<\epsilon<1<1}\left\{\int_{\partial \Omega}|u(\varphi(z))|^{p} d \sigma_{\epsilon}(z)\right\} \\
& =\limsup _{\epsilon \rightarrow 0^{+}}\left\{\int_{\partial \Omega}|u(\varphi(z))|^{p} d \sigma_{\epsilon}(z)\right\}=\int_{\Omega}|u(w)|^{p} d \nu(w) .
\end{aligned}
$$

Therefore, by the theorem of Hörmander [7], $C_{\varphi}: \mathscr{H}^{p}(\Omega) \rightarrow \mathscr{H}^{p}(\Omega)$ is bounded if and only if $d \nu$ is a Carleson measure. This completes the proof of (i).

Next we prove (ii). Suppose that $C_{\varphi}: \mathscr{H}^{p}(\Omega) \rightarrow \mathscr{H}^{p}(\Omega)$ is compact. We will show that $d \nu \in V C M(\Omega)$. Let $z_{0} \in \partial \Omega$, and let $C\left(z_{0}, r\right)$ be a Carleson region. Let $z \in \Omega$ be such that $\pi(z)=z_{0}$ and $r(z)=r$. Let $K(\cdot, \cdot)$ be the Bergman kernel for $\Omega$. Define a function

$$
k_{z, p}(w)=r(z)^{1 / p} K(z, z)^{-\gamma} K(w, z)^{2([1 / p]+1)} \quad(w \in \Omega),
$$

where $\gamma=2([1 / p]+1)-1 / p>0$ and $[x]$ denotes the largest integer not exceeding $x$. It is easy to check that $k_{z, p} \in \mathscr{H}^{p}(\Omega)$ and that, moreover, by using local coordinates and an estimate for the Bergman kernel in [8] for a strongly pseudoconvex domain (and [18] for a pseudoconvex domain of finite type in $\mathbf{C}^{2}$ with smooth boundary)

$$
\begin{aligned}
\left\|k_{z, p}\right\|_{\mathscr{R}^{p}}^{p} & =\sup _{0<\epsilon<<1} \int_{\partial \Omega_{\epsilon}} r(z) K(z, z)^{-\gamma p}|K(w, z)|^{2 p([1 / p]+1)} d \sigma_{\epsilon} \\
& \leq C_{p} r(z) K(z, z)^{-\gamma p} K(z, z)^{2 p([1 / p]+1)-1} r(z)^{-1} \leq C_{p} .
\end{aligned}
$$

It is clear that

$$
k_{z, p}(\cdot) \rightarrow 0 \quad \text { as } z \rightarrow \partial \Omega
$$

on compacta in $\Omega$. Since $C_{\varphi}: \mathscr{H}^{p}(\Omega) \rightarrow \mathscr{H}^{p}(\Omega)$ is compact, by Proposition 3.2 ,

$$
\left\|C_{\varphi}\left(k_{z, p}\right)\right\|_{\mathscr{R} P(\Omega)} \rightarrow 0 \quad \text { as } z \rightarrow \partial \Omega .
$$

Notice that, on the other hand,

$$
\begin{aligned}
\left\|C_{\varphi}\left(k_{p, z}\right)\right\|_{\mathscr{X} p(\Omega)}^{p} & =\int_{\Omega}\left|k_{z, p}(w)\right|^{p} d \nu(w) \\
& =r(z) K(z, z)^{-p \gamma} \int_{\Omega}|K(z, w)|^{2 p([1 / p]+1)} d \nu(w) \\
& \geq \frac{r(z)}{C_{p}\left|C\left(z_{0}, r\right)\right|} \int_{C\left(z_{0}, r\right)} d \nu(w) \geq \frac{1}{C_{p}\left|B\left(z_{0}, r\right)\right|} \int_{C\left(z_{0}, r\right)} d \nu(w) .
\end{aligned}
$$

Using this estimate, we shall show first that $d \nu \in B C M(\Omega)$ :

$$
\sup _{r} g_{\nu}(r)=\sup _{z_{0}, r}\left\{\frac{1}{\left|B\left(z_{0}, r\right)\right|} \int_{C\left(z_{0}, r\right)} d \nu\right\} \leq C_{p}\left\|C_{\varphi}\left(k_{p, z}\right)\right\|_{p}^{p} \leq C_{p}\left\|C_{\varphi}\right\|^{p}<\infty .
$$


Next we show that $d \nu \in V C M(\Omega)$. If this was not true, there would exist $z_{n}^{0} \in \partial \Omega, r_{n} \in(0,1)$ with $r_{n} \rightarrow 0$, and $\epsilon_{0}>0$ such that

$$
\frac{1}{\left|B\left(z_{n}^{0}, r_{n}\right)\right|} \int_{C\left(z_{n}^{0}, r_{n}\right)} d \nu \geq \epsilon_{0} \quad \text { for all } n \text {. }
$$

Now choose $z_{n} \in \Omega$ with $\pi\left(z_{n}\right)=z_{n}^{0}$ and $r\left(z_{n}\right)=r_{n}$. Then

$$
\epsilon_{0} \leq \frac{1}{\left|B\left(z_{n}^{0}, r_{n}\right)\right|} \int_{C\left(z_{n}^{0}, r_{n}\right)} d \nu \leq C_{p}\left\|C_{\varphi}\left(k_{p}, z_{n}\right)\right\|_{p}^{p} \quad \text { for all } n .
$$

Since $\left\{k_{p, z_{n}}\right\}$ is bounded in $\mathscr{H}^{p}, k_{p, z_{n}} \rightarrow 0$ on compacta as $n \rightarrow \infty$, and $C_{\varphi}$ is compact on $\mathscr{H}^{p}$, there is a subsequence $k_{p, z_{n_{k}}}$ such that $\left\|C_{\varphi}\left(k_{p, z_{n_{k}}}\right)\right\|_{\mathscr{C}_{p}} \rightarrow$ 0 . This contradiction completes the proof that $d \nu \in V C M(\Omega)$.

Now we show the converse. Let $d \nu \in V C M(\Omega)$. We will show $C_{\varphi}: \mathscr{H}^{p}(\Omega)$ $\rightarrow \mathscr{H}^{p}(\Omega)$ is compact. Suppose that $\left\{u_{n}\right\}$ is a bounded sequence in $\mathscr{H}^{p}(\Omega)$ which converges to 0 on compacta in $\Omega$. To show that

$$
C_{\varphi}\left(u_{n}\right) \rightarrow 0, \quad \text { in the metric of } \mathscr{H}^{p}(\Omega),
$$

let $\delta>0$ and set

$$
d \nu_{\delta}=\mathscr{Z}_{\Omega-\Omega_{\delta}} d \nu
$$

By using the covering lemma $[23,12]$, it follows that $d \nu_{\delta} \in B C M(\Omega)$ with

$$
\nu_{\delta}(C(z, r)) \leq g_{\nu}(\delta)|B(z, r)|, \quad g_{\nu}(\delta)=\sup _{x \in \partial \Omega}\left\{\frac{\nu(C(z, \delta))}{|B(z, \delta)|}\right\},
$$

for all $z \in \partial \Omega$ and $r>0$.

Since $d \nu \in V C M(\Omega), g_{\nu}(\delta) \rightarrow 0$ as $\delta \rightarrow 0$. Thus for every $\epsilon>0$, there is a $\delta>0$ such that $g_{\nu}(\delta)<\epsilon$. Since $u_{n} \rightarrow 0$ on compacta in $\Omega$, there is an $N$ such that for $n>N$,

$$
\int_{\Omega_{\delta}}\left|u_{n}(z)\right|^{p} d \nu(z)<\epsilon
$$

By (1),

$$
\begin{aligned}
\left\|C_{\varphi}\left(u_{n}\right)\right\|_{p}^{p} & =\int_{\Omega}\left|u_{n}(z)\right|^{p} d \nu(z) \\
& =\int_{\Omega_{\delta}}\left|u_{n}(z)\right|^{p} d \nu(z)+\int_{\Omega \backslash \Omega_{\delta}}\left|u_{n}(z)\right|^{p} d \nu(z) \\
& \leq \int_{\Omega_{\delta}}\left|u_{n}(z)\right|^{p} d \nu(z)+\int_{\Omega}\left|u_{n}(z)\right|^{p} d \nu_{\delta}(z) \\
& \leq \int_{\Omega_{\delta}}\left|u_{n}(z)\right|^{p} d \nu(z)+g_{\nu}(\delta) \int_{\partial \Omega}\left|u_{n}(z)\right|^{p} d \sigma(z) \\
& \leq \epsilon+\epsilon \sup \left\{\left\|u_{n}\right\|_{\mathscr{L} p}^{p}: n=1,2, \ldots\right\}
\end{aligned}
$$

when $n>N$. This completes the proof of sufficiency of (ii).

Now we prove (iii) and (iv). Note first that

$$
\int_{\partial \Omega}\left|C_{\varphi}(u)(z)\right|^{p} d \sigma(z)=\int_{\Omega}\left|P_{0}(u)(z)\right|^{p} d \nu(z)
$$

for all $u \in L^{p}(\sigma)$ and $p \geq 1$. Note next that since $\mathscr{H}^{p}(\Omega) \subset L^{p}(\partial \Omega)$ and $\mathscr{H}^{1}(\Omega) \subset \mathscr{H}^{1}(\partial \Omega)$, compactness of $C_{\varphi}$ on $L^{p}(\partial \Omega)$ or from $H^{1}(\partial \Omega)$ to 
$L^{1}(\partial \Omega)$ implies its compactness on $\mathscr{H}^{p}(\Omega)$ or $\mathscr{H}^{1}(\Omega)$, so $d \nu_{\varphi} \in V C M(\Omega)$, by (ii). Thus we need only show the sufficiency of (iii) and (iv).

In order to complete our proof, we need the following lemma, which can be obtained by using the fact that $P_{0}(z, w) \in C^{\infty}\left(\overline{\boldsymbol{\Omega}}_{\epsilon} \times \overline{\boldsymbol{\Omega}}\right)$ for any $\epsilon>0$, and the facts that $V M O(\partial \Omega)^{*}=H^{1}(\partial \Omega)$ and $L^{p^{\prime}}(\partial \Omega)=\left(L^{p}(\partial \Omega)\right)^{*}$ with $1 / p+1 / p^{\prime}=1$. We omit the detail here.

Lemma 3.3. Let $\left\{u_{n}\right\}$ be a bounded sequence in $H^{1}(\partial \Omega)$ or $L^{p}(\partial \Omega)$ with $1<$ $p<\infty$ such that $u_{n} \rightarrow 0$ in the $w^{*}$ topology. Then the sequence $\left\{P_{0}\left(u_{n}\right)\right\}$ converges to 0 on compacta in $\Omega$.

Now we continue our proof of sufficiency in (iii) and (iv). Here we present a proof of (iii). The proof of (iv) is similar and uses the duality $L^{p}(\partial \Omega)^{*}=$ $L^{p^{\prime}}(\partial \Omega)$ with $p$ and $p^{\prime}$ conjugate indices.

We have now reduced matters to the following lemma.

Lemma 3.4. Let $\Omega$ be a bounded strongly pseudoconvex domain in $\mathbf{C}^{n}$ with smooth boundary. Let $\varphi: \Omega \rightarrow \Omega$ be a holomorphic mapping.

(i) If $d \nu_{\varphi} \in B C M(\Omega)$, then $C_{\varphi}: H^{1}(\partial \Omega) \rightarrow L^{1}(\partial \Omega)$ is bounded.

(ii) If $d \nu_{\varphi} \in V C M(\Omega)$, then $C_{\varphi}: H^{1}(\partial \Omega) \rightarrow L^{1}(\partial \Omega)$ is compact.

Proof. Let $d \nu_{\varphi} \in B C M(\Omega)$ and $u \in H^{1}(\partial \Omega)$. It suffices to show that $C_{\varphi}(u) \in$ $L^{1}(\partial \Omega)$. Since

$$
\int_{\partial \Omega}\left|C_{\varphi}(u)(z)\right| d \sigma(z)=\int_{\Omega}\left|P_{0}(u)(z)\right| d \nu_{\varphi}(z)
$$

and since Hörmander's theorem holds for $P_{0}(u)$ with $u \in H^{1}(\partial \Omega)$ ([25]), we have

$$
\left\|C_{\varphi}(u)\right\|_{1} \leq C\|u\|_{H^{1}(\partial \Omega)},
$$

and (i) follows. Now we prove (ii). Suppose $d \nu \in V C M(\Omega)$. We shall prove that $C_{\varphi}: H^{1}(\partial \Omega) \rightarrow L^{1}(\partial \Omega)$ is compact. For each $0<\delta<<1$, let $d \nu_{\delta}$ be defined as above. Since $V M O(\partial \Omega)^{*}=H^{1}(\partial \Omega)$, it suffices to prove that for every (bounded) sequence $\left\{u_{n}\right\}$ in $H^{1}(\partial \Omega)$ with $u_{n} \rightarrow 0$ in the weak ${ }^{*}$ topology of $H^{1}(\partial \Omega)$, we have

$$
C_{\varphi}\left(u_{n}\right) \rightarrow 0 \text { in } L^{1}(\partial \Omega) \text {-norm. }
$$

Let $\epsilon>0$. By Lemma 3.3, there is an $N>1$ and $\delta>0$ such that if $n \geq N$

$$
\int_{\Omega_{\delta}}\left|P_{0}\left(u_{n}\right)(z)\right| d \nu(z)<\epsilon
$$

and

$$
g_{\nu}(\delta)<\epsilon
$$

By Hörmander's theorem again and the fact that $\nu_{\delta}(C(z, r)) \leq g_{\nu}(\delta)|B(z, r)|$ for any $z \in \partial \Omega$ and $r>0$, we have

$$
\left\|C_{\varphi}\left(u_{n}\right)\right\|_{1} \leq \epsilon+\epsilon \sup \left\{\left\|u_{n}\right\|_{H^{1}(\partial \Omega)}: n=1,2, \ldots\right\}
$$

when $n>N$. This proves Lemma 3.4, and therefore the proof of Theorem 3.1 is complete.

As a corollary of Theorem 3.1, we have the following result: 
Corollary 3.5. Let $\Omega$ be a bounded strongly pseudoconvex domain in $\mathbf{C}^{n}$ with smooth boundary. Let $\varphi: \Omega \rightarrow \Omega$ be a holomorphic mapping. Then:

(i) If there is a $0<p<\infty$ such that $C_{\varphi}: \mathscr{H}^{p}(\Omega) \rightarrow \mathscr{H}^{p}(\Omega)$ is bounded, then the measure $\left.d \nu_{\varphi}\right|_{\partial \Omega}$ is absolutely continuous with respect to surface measure $d \sigma$.

(ii) If $C_{\varphi}: \mathscr{H}^{p}(\Omega) \rightarrow \mathscr{H}^{p}(\Omega)$ is compact, then $\left.d \nu_{\varphi}\right|_{\partial \Omega}=0$.

(iii) If $C_{\varphi}: \mathscr{H}^{p}(\Omega) \rightarrow \mathscr{H}^{p}(\Omega)$ is bounded (resp. compact) for some $0<p<$ $\infty$, then it is bounded (resp. compact) for all $0<p<\infty$.

Proof. (i) Let $E$ be a set in $\partial \Omega$ such that $\sigma(E)=0$. We shall show that $\nu_{\varphi}(E)=0$. For any $\epsilon>0$, there is a sequence of nonisotropic balls $\left\{B\left(z_{j}, r_{j}\right)\right\}$ on $\partial \Omega$ such that

$$
E \subset \bigcup_{j=1}^{\infty} B\left(z_{j}, r_{j}\right), \quad \text { and } \quad \sum_{j=1}^{\infty} \sigma\left(B\left(z_{j}, r_{j}\right)\right) \leq \epsilon .
$$

Let $C\left(z_{j}, r_{j}\right)$ be Carleson regions for $j=1,2, \ldots$. Then

$$
\begin{aligned}
\nu_{\varphi}(E) & \leq \nu_{\varphi}\left(\bigcup_{j=1}^{\infty} B\left(z_{j}, r_{j}\right)\right) \leq \sum_{j=1}^{\infty} \nu_{\varphi}\left(B\left(z_{j}, r_{j}\right)\right) \leq \sum_{j=1}^{\infty} \nu_{\varphi}\left(C\left(z_{j}, r_{j}\right)\right) \\
& \leq \sum_{j=1}^{\infty} C \sigma\left(B\left(z_{j}, r_{j}\right)\right) \leq C \epsilon .
\end{aligned}
$$

Since $\epsilon$ is arbitrary, (i) follows, and (ii) can be obtained by using an argument similar to the proof of (i) and the covering lemma. Since (iii) is a direct consequence of Theorem 3.1, the proof of Corollary 3.5 is complete.

\section{Proof of the MAIN THeOREM}

In this section, we shall complete the proof of Theorem 1. As noted earlier, by Theorem 3.1, (ii), (iii), (iv), (v), (vi), and (vii) are equivalent. We shall show that (i) is equivalent to (ii). Half of this statement is contained in the next result.

Theorem 4.1. Let $\Omega$ be bounded strongly pseudoconvex domain in $\mathbf{C}^{n}$ with smooth boundary. Let $\varphi: \Omega \rightarrow \Omega$ be a holomorphic mapping. If $C_{\varphi}^{*}: L^{\infty}(\partial \Omega) \rightarrow$ $V M O(\partial \Omega)$ is bounded, then

(a) $\left.d \nu_{\varphi}\right|_{\partial \Omega}=0$, and

(b) $C_{\varphi}: H^{1}(\partial \Omega) \rightarrow L^{1}(\partial \Omega)$ is compact.

Proof. Since $C_{\varphi}: H^{1}(\partial \Omega) \rightarrow L^{1}(\partial \Omega)$ is bounded and $\varphi$ is holomorphic, it follows from Corollary 3.5 (i) that $\left.d \nu_{\varphi}\right|_{\partial \Omega}$ is absolutely continuous with respect to $\sigma$, with nonnegative Radon-Nikodým derivative $g=\left(\left.d \nu_{\varphi}\right|_{\partial \Omega}\right) / d \sigma \in L^{1}(\sigma)$.

Suppose the assertion (a) is not true. Then there is a Borel set $G \subset \partial \Omega$ and $\epsilon>0$ such that $0<\sigma(G)<<1$ and $\frac{1}{\epsilon} \geq g(z)>\epsilon>0$ on $G$. For each $0<\delta<<1$, we choose $z_{\delta} \in \partial \Omega$ so that $\left|B\left(z_{\delta}, \delta\right) \cap G\right|=\alpha(\delta)|G|\left|B\left(z_{\delta}, \delta\right)\right|$, where $\alpha(\delta)$ is a positive constant satisfying $\frac{1}{C(n)} \leq \alpha(\delta) \leq 1$ and $C(n)$ is a constant depending only on $n$.

Let $F=\varphi^{-1}(G)$. Then for any $h \in L^{\infty}(\partial \Omega)$, we claim that

$$
C_{\varphi}^{*}\left((h \circ \varphi) \mathscr{X}_{F}\right)=h g \mathscr{X}_{G}, \quad \text { on } \partial \Omega \text {. }
$$


To see this, let $u \in L^{1}(\partial \Omega)$. Then

$$
\begin{aligned}
\left\langle u, C_{\varphi}^{*}\left((h \circ \varphi) \mathscr{Z}_{F}\right)\right\rangle & =\left\langle C_{\varphi}(u),(h \circ \varphi) \mathscr{X}_{F}\right\rangle=\int_{F} P_{0}(u)(\varphi(z)) \overline{h(\varphi(z))} d \sigma(z) \\
& =\int_{\partial \Omega}\left(P_{0}(u) \circ \varphi\right)(\overline{h \circ \varphi})\left(\mathscr{X}_{G} \circ \varphi\right) d \sigma=\int_{\bar{\Omega}} P_{0}(u) \bar{h} \mathscr{X}_{G} d \nu_{\varphi} \\
& =\int_{\partial \Omega} u \bar{h} \mathscr{X}_{G} g d \sigma=\left\langle u, g h \mathscr{X}_{G}\right\rangle .
\end{aligned}
$$

If we now let

$$
h(z)= \begin{cases}1 / g(z) & \text { if } z \in G, \\ 0 & \text { if } z \in \partial \Omega \backslash G\end{cases}
$$

then $h g=\mathscr{X}_{G}$, and from the above claim and the fact that $C_{\varphi}^{*}: L^{\infty}(\partial \Omega) \rightarrow$ $V M O(\partial \Omega)$ is bounded we conclude that $\mathscr{X}_{G} \in V M O(\partial \Omega)$.

To complete the proof, we shall show that $\mathscr{Z}_{G}$ is not in $V M O(\partial \Omega)$. In what follows, let $B=B\left(z_{\delta}, \delta\right)$. Since

$$
\frac{1}{|B|} \int_{B} \mathscr{X}_{G} d \sigma=|G \cap B| /|B|=\alpha(\delta)|G|,
$$

we have

$$
\begin{aligned}
& \frac{1}{|B|} \int_{B}\left|\mathscr{X}_{G}(z)-\frac{1}{|B|} \int_{B} \mathscr{X}_{G} d \sigma\right| d \sigma(z) \\
& \quad=\frac{1}{|B|} \int_{B}\left|\mathscr{X}_{G}(z)-\frac{|B \cap G|}{|B|}\right| d \sigma(z) \geq \frac{1}{|B|} \int_{B \cap G}(1-\alpha(\delta)|G|) d \sigma(z) \\
& \quad=\frac{1}{|B|}(1-\alpha(\delta)|G|)|G \cap B| \geq \frac{1}{2} \alpha(\delta)|G| \geq \frac{1}{2 C(n)}|G| .
\end{aligned}
$$

Therefore, $\mathscr{X}_{G} \notin V M O(\Omega)$, and the proof of $(\mathrm{a})$ is complete.

Now we prove (b). Since $V M O(\partial \Omega)^{*}=H^{1}(\partial \Omega)$, it suffices to prove that for every bounded sequence $\left\{u_{n}\right\} \subset H^{1}(\partial \Omega)$ which converges to 0 in the weak * topology of $H^{1}(\partial \Omega)$, it follows that $C_{\varphi}\left(u_{n}\right)$ converges to 0 in $L^{1}(\partial \Omega)$-norm. Let $\left\{u_{n}\right\}$ be such a sequence. By part $(\mathrm{a}), \varphi(z) \in \Omega$ for $\sigma$-a.e. $z \in \partial \Omega$, and thus by Lemma 3.3, $C_{\varphi}\left(u_{n}\right)(z) \rightarrow 0$ for $\sigma$-a.e. $z \in \partial \Omega$. But $C_{\varphi}\left(u_{n}\right) \rightarrow 0$ weakly in the topology of $L^{1}(\partial \Omega)$. To see this, note that for $g \in L^{\infty}(\partial \Omega)$

$$
\left\langle C_{\varphi}\left(u_{n}\right), g\right\rangle=\left\langle u_{n}, C_{\varphi}^{*}(g)\right\rangle \rightarrow 0
$$

as $n \rightarrow \infty$ since $C_{\varphi}^{*}(g) \in V M O(\partial \Omega)$ and $V M O(\partial \Omega)^{*}=H^{1}(\partial \Omega)$. Now by [6, p. 295], $\left\|C_{\varphi}\left(h_{n}\right)\right\|_{L^{1}(\partial \Omega)} \rightarrow 0$ as $n \rightarrow \infty$ and (b) follows. The proof of Theorem 4.1 is complete.

We now finish the proof of Theorem 1. As noted above, by Theorem 4.1, (i) implies (ii). Next we show that (ii) implies (i). Let $g \in L^{\infty}(\partial \Omega)$. We show that $C_{\varphi}^{*}(g) \in V M O(\partial \Omega)$. Since we are assuming $C_{\varphi}: H^{1}(\partial \Omega) \rightarrow L^{1}(\partial \Omega)$ is compact, it is obvious that $C_{\varphi}^{*}: L^{\infty}(\partial \Omega) \rightarrow B M O(\partial \Omega)$ is bounded, so we need only show that $C_{\varphi}^{*}(g) \in V M O(\partial \Omega)$. Notice again that $V M O(\partial \Omega)^{*}=H^{1}(\partial \Omega)$ and that $V M O(\partial \Omega)$ is a separable Banach space. So by Corollary V.12.8 in [4], $C_{\varphi}^{*}(g) \in V M O(\partial \Omega)$ if for each sequence $\left\{u_{n}\right\} \subset H^{1}(\partial \Omega)$ which converges to 0 in the weak ${ }^{*}$ topology of $H^{1}(\partial \Omega)$ it follows that $\left\langle C_{\varphi}^{*}(g), u_{n}\right\rangle \rightarrow 0$ as $n \rightarrow \infty$. Now let $\left\{u_{n}\right\}$ be such a sequence in $H^{1}(\partial \Omega)$. By the Uniform Boundedness 
Theorem, $\left\{u_{n}\right\}$ is a bounded sequence in $H^{1}(\partial \Omega)$. Suppose it is not true that $\left\langle C_{\varphi}^{*}(g), u_{n}\right\rangle \rightarrow 0$ as $n \rightarrow \infty$. Then there is a subsequence, we use the same notation, $\left\{u_{n}\right\}$ and $\epsilon_{0}>0$ so that

$$
\left|\left\langle g, C_{\varphi}\left(u_{n}\right)\right\rangle\right|=\left|\left\langle C_{\varphi}^{*}(g), u_{n}\right\rangle\right| \geq \epsilon_{0}
$$

for all $n$. Since $C_{\varphi}: H^{1}(\partial \Omega) \rightarrow L^{1}(\partial \Omega)$ is compact, there is a subsequence $\left\{u_{n_{k}}\right\}$ and $v \in L^{1}(\partial \Omega)$ such that $C_{\varphi}\left(u_{n_{k}}\right) \rightarrow v$ in $L^{1}(\partial \Omega)$-norm. We shall show that $v=0$, contradicting (2). Since $u_{n} \rightarrow 0$ in the weak* topology of $H^{1}(\partial \Omega), P_{0}\left(u_{n}\right) \rightarrow 0$ on compacta in $\Omega$ by Lemma 3.3. Since (ii) implies (iv) in Theorem 1 has already been proved, it follows from (ii) in Corollary 3.5 that

$$
C_{\varphi}\left(u_{n}\right)(z)=P_{0}\left(u_{n}\right)(\varphi(z)) \rightarrow 0, \quad \text { for a.e. } z \in \partial \Omega
$$

as $n \rightarrow \infty$. Therefore, $v=0$. The proof of Theorem 1 is now complete.

\section{REFERENCES}

1. J. A. Cima and W. Wogen, Unbounded composition operators on $H^{2}\left(B_{n}\right)$, Proc. Amer. Math. Soc. 99 (1987), 477-483.

2. R. R. Coifman and G. Weiss, Extensions of Hardy spaces and their use in analysis, Bull. Amer. Math. Soc. 83 (1977), 569-643.

3. M. Christ and D. Geller, Singular integral characterizations of Hardy spaces on homogeneous groups, Duke Math. J. 51 (1984), 547-598.

4. J. B. Conway, A course in functional analysis, 2nd ed., Springer-Verlag, New York, 1990.

5. C. C. Cowen, Composition operators on Hilbert spaces of analytic functions: A status report, Proc. Sympos. Pure Math., vol. 51, Amer. Math. Soc., Providence, RI, 1990, pp. 131-145.

6. N. Dunford and J. T. Schwartz, Linear operator, Part I, Interscience, New York, 1958.

7. L. Hörmander, $L^{p}$-estimates for pluri-harmonic functions, Math. Scand. 20 (1967), 65-78.

8. C. Fefferman, The Bergman kernel and biholomorphic mappings of pseudoconvex domains, Invent. Math. 26 (1974), 1-65.

9. C. Fefferman and E. M. Stein, $H^{p}$ spaces of several variables, Acta Math. 129 (1972), 137-193.

10. F. Jafari, Carleson measures in Hardy and weighted Bergman spaces of poly discs, Proc. Amer. Math. Soc. 112 (1991), 771-781.

11. N. Kerzman, The Bergman kernel function. Differentiability at the boundary, Math. Ann. 195 (1972), 149-158.

12. S. G. Krantz, Function theory of several complex variables, 2nd ed., Wadsworth, Belmont, CA, 1992.

13. S. G. Krantz and S.-Y. Li, A note on Hardy spaces and functions of bounded mean oscillation on domains in $\mathbf{C}^{n}$, Michigan Math. J. (to appear).

14. _ On decomposition theorems for Hardy spaces on domains in $\mathbf{C}^{n}$ and applications, preprint.

15. B. MacCluer, Spectra of composition operators on $H^{p}\left(B_{N}\right)$, Analysis 4 (1984), 87-103.

16. Compact composition operators on $H^{p}\left(B_{N}\right)$, Michigan Math. J. 32 (1985), 237-248.

17. A. Nagel, E. M. Stein, and S. Wainger, Boundary behavior of functions holomorphic in domains of finite type, Proc. Nat. Acad. Sci. U.S.A. 78 (1981), 6596-6599.

18. A. Nagel, J. P. Rosay, E. M. Stein, and S. Wainger, Estimates for the Bergman and Szegö kernels in $\mathbf{C}^{2}$, Ann. of Math. (2) 129 (1989), 113-149.

19. J. H. Shapiro, The essential norm of a composition operator, Ann. of Math. (2) 125 (1987), 375-404.

20. D. Sarason, Weak compactness of holomorphic composition operators on $H^{1}$, preprint. 
21. J. H. Shapiro and C. Sundberg, Compact composition operators on $L^{1}$, Proc. Amer. Math. Soc. 108 (1992), 443-449.

22. J. H. Shapiro and P. D. Taylor, Compact nuclear, and Hilbert Schmidt composition operators on $H^{2}$, Indiana Univ. Math. J. 23 (1973), 471-496.

23. E. M. Stein, Boundary behavior of holomorphic functions of several complex variables, Princeton Univ. Press, Princeton, NJ, 1972.

24. W. Wogen, Composition operators acting on spaces of holomorphic functions on domains in $\mathbf{C}^{n}$, Proc. Sympos. Pure Math., vol. 51, Amer. Math. Soc., Providence, RI, 1990, pp. 361-366.

25. R. R. Coifman, Y. Meyer, and E. M. Stein, Some new function spaces and their application to harmonic analysis, J. Funct. Anal. 62 (1985), 304-335.

26. B. MacCluer and J. H. Shapiro, Angular derivatives and compact composition operators on the Hardy and Bergman spaces, Canad. J. Math. 38 (1986), 878-906.

Department of Mathematics, University of California at Irvine, Irvine, California 92717

Current address, Song-Ying Li: Department of Mathematics, Washington University, St. Louis, Missouri 63130

Current address, Bernard Russo: Department of Mathematics, University of California, Irvine, California 92717

E-mail address, B. Russo: brusso@math.uci.edu 